\title{
Therapeutic applications and antibacterial and antifungal activities of Syzygium cumini (L.) Skeels extracts: a systematized literature review focusing on dental applications
}

Aplicações terapêuticas, atividades antibacteriana e antifúngica de extratos de Syzygium cumini (L.) Skeels: uma revisão sistematizada da literature com ênfase em suas aplicações odontológicas

Aplicaciones terapéuticas, actividades antibacteriana y antifúngica de extractos de Syzygium cumini (L.) Skeels: una revisión sistematizada con énfasis en sus aplicaciones odontológicas

\author{
Ernani Canuto FIGUEIRÊDO JÚNIOR ${ }^{1}$ \\ Bruna Palmeira COSTA ${ }^{1}$ \\ Julliana Cariry Palhano FREIRE ${ }^{1}$ \\ Waleska Ohana de Souza MELO \\ Cristina Ruan FERREIRA DE ARAÚJO' \\ Edja Maria Melo de Brito COSTA ${ }^{3}$ \\ Jozinete Vieira PEREIRA ${ }^{3}$
}

${ }^{1}$ Postgraduate Program in Dentistry, State University of Paraíba, UEPB 58429-500, Campina Grande, PB, Brazil

${ }^{2}$ Science Center Biological and Health, Federal University of Campina Grande, UFCG 58429-900, Campina Grande, PB, Brazil

${ }^{3}$ Department of Dentistry, State University of Paraíba, UEPB 58429-500, Campina Grande, PB, Brazil

\section{Abstract}

Medicinal plants are an important source of curative substances for the treatment of diseases. Investigations are focusing particularly on medicinal plants used to treat affections of the oral cavity. Among these species, there is evidence of potential applications of Syzygium cumini to Dentistry. The objective of this systematized literature review was to synthesize the evidence on the antimicrobial activity of this plant, as well cite some as the traditional uses of its extracts for dental purposes. The data indicate that the extracts prepared from different parts of the plant have antibacterial and antifungal effects on different pathogenic microorganisms of the oral microbiota and/or those that can potentially cause oral diseases, demonstrating a promising therapeutic potential for indication and use in Dentistry to prevent and/or treat oral affections.

Descriptors: Plant Extracts; Medicine, Traditional; Anti-Bacterial Agents; Antifungal Agents; Dentistry.

\section{Resumo}

As plantas medicinais representam uma fonte importante para a obtenção de substâncias com fins curativos no tratamento de doenças. Dentre essas, tem se destacado a investigação daquelas voltadas para o tratamento de afecções que acometem a cavidade bucal. Dentre essas espécies, Syzygium cumini tem apresentado evidências com potencial aplicação e utilização na Odontologia. Desse modo, o presente trabalho tem como objetivo sintetizar através de uma revisão sistematizada da literatura as evidências acerca da atividade antimicrobiana, bem como dos usos populares de seus extratos com interesse odontológico. As pesquisas foram realizadas nas bases de dados National Library of Medicine National Institutes of Health (PubMed), Scientific Electronic Library Online (Scielo) e Google Scholar. Os dados apontam que os extratos de diferentes partes da planta apresentam efeitos antibacterianos e antifúngicos sobre diversos microrganismos patogênicos presentes e/ou potencialmente causadores de doenças bucais, demonstrando um potencial terapêutico promissor para sua indicação e utilização odontológica na prevenção e/ou o tratamento de afecões bucais.

Descritores: Extratos Vegetais; Medicina Tradicional; Antibacterianos; Antifúngicos; Odontologia.

\section{Resumen}

Las plantas medicinales representan una fuente importante para la obtención de sustancias con fines curativos en el tratamiento de enfermedades. Entre ellas, se ha destacado la investigación de aquellas orientadas hacia el tratamiento de afecciones que acomete la cavidad bucal. Entre estas especies, Syzygium cumini ha presentado evidencias con potencial aplicación y utilización en la Odontología. De este modo, el presente trabajo tiene como objetivo sintetizar a través de una revisión sistematizada de la literatura las evidencias acerca de la actividad antimicrobiana, así como de algunos de los usos populares de sus extractos con interés odontológico. Los datos apuntan que los extractos de diferentes partes de la planta presentan efectos antibacterianos y antifúngicos sobre diversos microorganismos patógenos presentes y/o potencialmente causantes de enfermedades bucales, demostrando un potencial terapéutico prometedor para su indicación y uso odontológico en la prevención y/o el tratamiento de los afecones la boca.

Descriptores: Extractos Vegetales; Medicina Tradicional; Antibacterianos; Antifúngicos; Odontología.

INTRODUCTION

The use of medicinal plants for curative purposes is intrinsically related to the early days of medicine when they were used as a basis in the treatment of various diseases ${ }^{1,2}$. Medicinal plants have been for a long time the primary source of material for the production and development of drugs ${ }^{3}$. Although medicine is at an advanced technical and scientific stage in most parts of the world, $80 \%$ of the population of developing countries continues to primarily rely on traditional resources and practices for basic health care $e^{4-6}$.

Within this context, given the increasing acceptance of phytotherapy as an alternative therapeutic modality ${ }^{7,8}$, research is focusing particularly on medicinal plants with applications to Dentistry because of the large number of species that have been reported for the treatment of affections of the oral cavity ${ }^{8,9}$. Among these species, the plant Syzygium cumini (L.) Skeels (Myrtaceae) (synonyms: Syzygium jambolanum, Syzygium jambolana, Eugenia jambolana, Eugenia cumini) ${ }^{1,10-12}$ commonly known as jambolão, jamun, azeitona, azeitona-roxa and olive ${ }^{1,10,11,13}$, is used in folk medicine because of its different pharmacological properties $\mathrm{s}^{1,3,10-18}$.

In this scenario, considering the need for studies investigating medicinal plants with potential application to Dentistry ${ }^{8,9}$, the objective of this literature review was to synthesize the biological activities of potential interest for 
Dentistry of $S$. cumini, focusing on its antimicrobial and antifungal effects on microorganisms of the oral microbiota.

MATERIAL AND METHOD

This study consists of a literature review and bibliometric study of original articles evaluating the antibacterial and antifungal activities of $S$. cumini extracts on microorganisms of the oral microbiota and/or microorganisms that can potentially cause oral diseases, focusing on some uses and indications of these extracts with potential interest to Dentistry.

\section{- Search strategy}

For the literature review and bibliometric study, searches were performed in May 2018 using the National Library of Medicine National Institutes of Health (PubMed), Scientific Electronic Library Online (Scielo) and Google Scholar databases. Articles without publication date and language restrictions were selected. The following terms were used: "Syzygium cumini", "antifungal activity", "antimicrobial activity" through the search strategy using Boolean operators "AND" and "OR" as follows: "(Syzygium cumini) AND (antimicrobial activity OR antifungal activity)".

\section{- Inclusion and exclusion criteria}

Only complete literature reviews and experimental studies addressing the topic proposed and that had only used extracts from different parts of $S$. cumini were included for fulltext reading. Studies investigating compounds isolated from the plant or synthesized compounds were not considered. Incomplete articles, monographs, master's dissertations, doctoral theses and publications not consistent with the topic were excluded. Articles investigating $S$. cumini extracts combined with other substances were also eliminated.

\section{- Study selection}

Analysis and selection of the retrieved articles were performed in three steps. In the first step, the title of the articles was read and those whose title indicated studies related to the antimicrobial and antifungal potential of $S$. cumini extracts against oral microorganisms, as well as dental applications, were selected. The second step consisted of reading the abstracts of the selected articles. Finally, the third step consisted of full-text reading of the articles and qualitative analysis of the studies that addressed the topic proposed and met the eligibility criteria. Studies reporting some evidence on the subject of this investigation were selected.
RESULTS

The searches retrieved 5.337 articles, being 260 from PubMed, seven from Scielo and 5.110 from Google Scholar, respectively. After analysis of the title, 1.084 article were excluded and after this step, the abstracts of 174 articles were read and those that addressed the topic proposed and met the eligibility criteria were selected for full-text reading (61 articles). After exclusion of duplicated articles, evaluation and qualitative analysis of the articles retrieved a final sample of 42 articles, including studies related to the antibacterial and antifungal activities of $S$. cumini extracts against microorganisms of the oral microbiota and/or microorganisms that can potentially cause oral diseases (Figure 1).

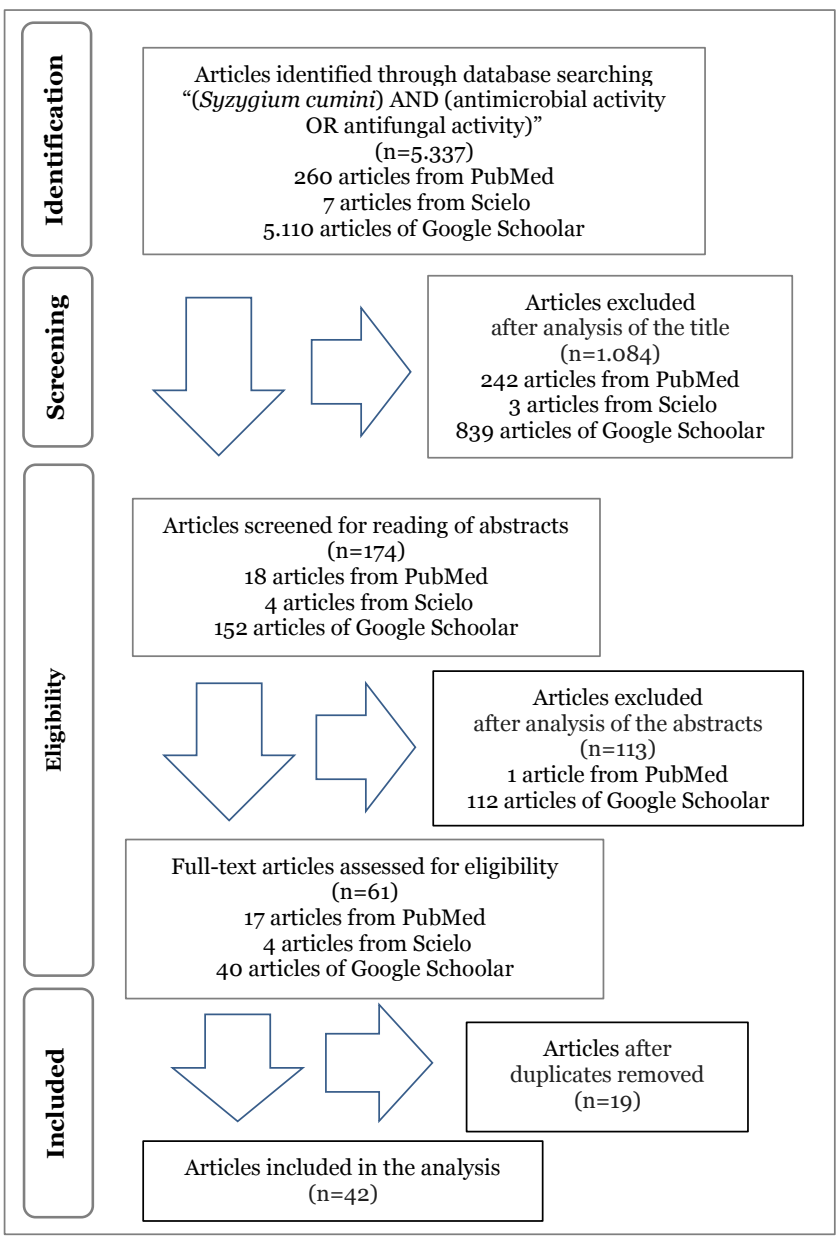

Figure 1: Research data flowchart

Some findings about the popular use of S. cumini for therapeutic purposes and application of $S$. cumini extracts to Dentistry is described in Table 1. Evidence available in the literature on the antibacterial and antifungal effects attributed to the $S$. cumini extracts on microorganisms of the oral microbiota and/or microorganisms that can potentially cause diseases of the oral cavity is described in Tables 2 and 3 , respectively. 
Table 1. Therapeutic use and application of different parts of Syzygium

\begin{tabular}{|c|c|c|c|}
\hline $\begin{array}{l}\text { Plant } \\
\text { parts }\end{array}$ & Uses & Therapeutic purposes & References \\
\hline \multirow[t]{2}{*}{ Fruits } & Gargles & Treatment of throat irritation & Migliato et al. ${ }^{1}$ (2006) \\
\hline & ${ }^{*} \mathrm{NS}$ & Treatment of halitosis & $\begin{array}{l}\text { Ayynamar \& Subash-Babu }{ }^{10} \\
\text { (2012) }\end{array}$ \\
\hline \multirow[t]{2}{*}{ Leaves } & ${ }^{*} \mathrm{NS}$ & $\begin{array}{l}\text { Strengthening effects on teeth and } \\
\text { gingiva }\end{array}$ & $\begin{array}{l}\text { Ayynamar \& Subash-Babu }{ }^{10} \\
\text { (2012); Srivastava \& Chandra }{ }^{11} \\
\text { (2013); Atale et al. }{ }^{14}(2013) ; \\
\text { Gowri \& Vasantha }{ }^{22}(2010) ; \\
\text { Borde et al. }{ }^{23}(2013)\end{array}$ \\
\hline & Mouthwash & $\begin{array}{l}\text { Treatment of recurrent aphthous } \\
\text { ulcers, stomatitis, affections of the } \\
\text { throat and other oral diseases }\end{array}$ & Costa et al. ${ }^{21}(2009)$ \\
\hline Seeds & Extract & $\begin{array}{l}\text { Treatment of oral eruptions and } \\
\text { throat }\end{array}$ & $\begin{array}{l}\text { Ayynamar \& Subash-Babu }{ }^{10} \\
\text { (2012); Chandrasekaran \& } \\
\text { Venkatesalu }{ }^{24}(2004)\end{array}$ \\
\hline \multirow[t]{3}{*}{ Stem } & ${ }^{*} \mathrm{NS}$ & $\begin{array}{l}\text { Antiseptic, astringent of oral } \\
\text { ulcerations and stomatitis }\end{array}$ & Migliato et al. ${ }^{1}(2006)$ \\
\hline & $\begin{array}{l}\text { Decoction as } \\
\text { mouthwash }\end{array}$ & $\begin{array}{l}\text { Treatment of recurrent aphthous } \\
\text { ulcers, stomatitis and affections of } \\
\text { the throat }\end{array}$ & Loguercio et al. ${ }^{19}(2005)$ \\
\hline & $\begin{array}{l}\text { Decoction as } \\
\text { mouthwash }\end{array}$ & $\begin{array}{l}\text { Astringent effect on oral } \\
\text { ulcerations, gingival hyperplasia } \\
\text { and stomatitis }\end{array}$ & Ulla et al. ${ }^{20}$ (2017) \\
\hline
\end{tabular}

Legend: *NS= not specified.

Table 2. Evidence of studies demonstrating antibacterial activity of Syzygium cumini extracts obtained from different parts of the plant against microorganisms of interest to Dentistry.

\begin{tabular}{|c|c|c|c|}
\hline $\begin{array}{l}\text { Plant } \\
\text { parts }\end{array}$ & Microorganisms & $\begin{array}{c}\text { Antimicrobial } \\
\text { susceptibility test }\end{array}$ & References \\
\hline \multirow[t]{4}{*}{ Fruits } & Bacillus subtilis & Disk diffusion & Saha et al. ${ }^{25}$ (2013) \\
\hline & Escherichia coli & $\begin{array}{l}\text { Agar dilution } \\
\text { Disk diffusion }\end{array}$ & $\begin{array}{l}\text { Shad et al. }{ }^{26}(2014) \\
\text { Saha et al. }{ }^{25}(2013)\end{array}$ \\
\hline & $\begin{array}{l}\text { Pseudomonas } \\
\text { aeruginosa }\end{array}$ & $\begin{array}{l}\text { Agar dilution } \\
\text { Microdilution }\end{array}$ & $\begin{array}{c}\text { Shad et al. }{ }^{26}(2014) \\
\text { Migliato et al. }{ }^{27}(2010)\end{array}$ \\
\hline & Staphylococcus aureus & $\begin{array}{l}\text { Disk diffusion } \\
\text { Microdilution }\end{array}$ & $\begin{array}{c}\text { Saha et al. }{ }^{25}(2013) \\
\text { Migliato et al. }{ }^{27}(2010)\end{array}$ \\
\hline $\begin{array}{l}\text { Fruit } \\
\text { peel }\end{array}$ & $\begin{array}{l}\text { Enterococcus faecalis; } \\
\text { Escherichia } \\
\text { coli;Pseudomonas } \\
\text { aeruginosa; } \\
\text { Staphylococcus aureus }\end{array}$ & $\begin{array}{l}\text { Disk diffusion; } \\
\text { Macrodilution }\end{array}$ & Priya et al. ${ }^{28}(2013)$ \\
\hline \multirow[t]{11}{*}{ Leaves } & Bacillus subtilis & $\begin{array}{l}\text { Disk diffusion } \\
\text { Microdilution }\end{array}$ & $\begin{array}{c}\text { Kaneria et al. }{ }^{5}(2009) \text {; Gowri \& } \\
\text { Vasantha }{ }^{22}(2010) ; \text { Shafi } \text { et al. }{ }^{29} \\
\text { (2002); Kaneria \& Chanda }{ }^{11} \\
(2011) ; \text { Tahir et al. }{ }^{33}(2012) ; \\
\text { Mohamed et al. }{ }^{34}(2013) ; \text { Elfadil } \\
\text { et al. }{ }^{35}(2015) \\
\text { Bhargava et al. }{ }^{30}(2009) ; \\
\text { Elansary et al. }{ }^{32}(2012) \\
\text { Elansary et al. }{ }^{32}(2012)\end{array}$ \\
\hline & Enterococcus faecalis & $\begin{array}{l}\text { Agar diffusion } \\
\text { Microdilution }\end{array}$ & $\begin{array}{l}\text { Oliveira et al. }{ }^{8}(2007) ; \\
\text { Mohamed et al. }{ }^{34(2013)} \\
\text { Pereira et al. }{ }^{9}(2009)\end{array}$ \\
\hline & Escherichia coli & $\begin{array}{l}\text { Disk diffusion } \\
\text { Microdilution }\end{array}$ & 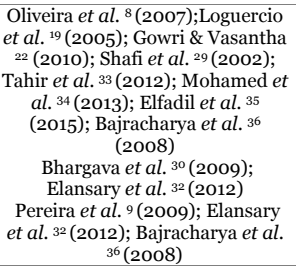 \\
\hline & Klebsiella pneumoniae & $\begin{array}{l}\text { Agar diffusion } \\
\text { Disk diffusion }\end{array}$ & $\begin{array}{l}\text { Kaneria \& Chanda }{ }^{31}(2011) ; \\
\text { Bouzada et al. }{ }^{37}(2009) \\
\text { Bhargava et al. }{ }^{30}(2009)\end{array}$ \\
\hline & Neisseria gonorrhoeae & $\begin{array}{l}\text { Agar diffusion } \\
\text { Disk diffusion } \\
\text { Microdilution }\end{array}$ & $\begin{array}{c}\text { Oliveira et al. }{ }^{8}(2007) \text {; Jadhav } \\
\text { al. } 3^{8}(2015) \\
\text { Mohamed et al. }{ }^{44}(2013) \\
\text { Jadhav al. }{ }^{8}(2015)\end{array}$ \\
\hline & $\begin{array}{l}\text { Pseudomonas } \\
\text { aeruginosa }\end{array}$ & $\begin{array}{l}\text { Disk diffusion; } \\
\text { Microdilution }\end{array}$ & $\begin{array}{l}\text { Kaneria et al. } 5 \text { (2009); Oliveira et } \\
\text { al. }{ }^{8}(2007) ; \text { Loguercio et al. }{ }^{19} \\
\text { (2005); Gowri \& Vasantha }{ }^{22} \\
\text { (2010); Shafi et al. }{ }^{29}(2002) ; \\
\text { Tahir et al. }{ }^{33}(2012) ; \text { Mohamed et } \\
\text { al. }{ }^{34}(2013) ; \text { Elfadil et al. } 35 \\
\text { (2015); Bouzada et al. } .^{37}(2009) \\
\text { Elansary et al. }{ }^{32}(2012) \\
\text { Elansary et al. }{ }^{32}(2012)\end{array}$ \\
\hline & Streptococcus mutans & $\begin{array}{l}\text { Agar diffusion } \\
\text { Macrodilution } \\
\text { Microdilution }\end{array}$ & $\begin{array}{c}\text { Tahir et al. }{ }^{33}(2012) \text {; Viera et al. } \\
\text { Vie (2012) } \\
\text { Viera et al. } 39(2012) \\
\text { Pereira et al. }{ }^{9}(2009)\end{array}$ \\
\hline & $\begin{array}{l}\text { Lactobacillus casei; } \\
\text { Streptococcus oralis; } \\
\text { Streptococcus } \\
\text { parasanguis; } \\
\text { Streptococcus salivarius }\end{array}$ & $\begin{array}{l}\text { Agar diffusion; } \\
\text { Macrodilution }\end{array}$ & Viera et al. ${ }^{39}$ (2012) \\
\hline & Streptococcus viridans & Agar diffusion & Tahir et al. 33 (2012) \\
\hline & Staphylococcus aureus & $\begin{array}{l}\text { Disk diffusion } \\
\text { Macrodilution } \\
\text { Microdilution }\end{array}$ & 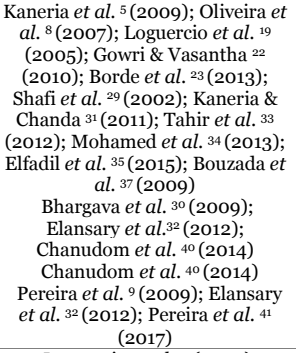 \\
\hline & $\begin{array}{l}\text { Staphylococcus } \\
\text { intermedius }\end{array}$ & Agar diffusion & Loguercio et al. ${ }^{19}$ (2005) \\
\hline
\end{tabular}

Table 2 - Continuation. Evidence of studies demonstrating antibacterial activity of Syzygium cumini extracts obtained from different parts of the plant against microorganisms of interest to Dentistry.

\begin{tabular}{|c|c|c|c|}
\hline $\begin{array}{l}\text { Plant } \\
\text { parts }\end{array}$ & Microorganisms & $\begin{array}{c}\text { Antimicrobial } \\
\text { susceptibility test }\end{array}$ & References \\
\hline \multirow[t]{6}{*}{ Seeds } & Bacillus subtilis & $\begin{array}{l}\text { Agar diffusion } \\
\text { Disk diffusion; } \\
\text { Macrodilution } \\
\text { Microdilution }\end{array}$ & $\begin{array}{c}\text { Chandrasekaran \& Venkatesalu } 24 \\
\text { (2004); Yadav et al. } 42 \text { (2011); Yadav } \\
\text { et al. } 43 \text { (2017) } \\
\text { Duraipandiyan et al. } 4 \text { (2006);Saha } \\
\text { et al. } 25 \text { (2013) } \\
\text { Chandrasekaran \& Venkatesalu } 24 \\
\text { (2004) } \\
\text { Yadav et al. } 43 \text { (2017) }\end{array}$ \\
\hline & Escherichia coli & $\begin{array}{l}\text { Disk diffusion } \\
\text { Macrodilution } \\
\text { Microdilution }\end{array}$ & $\begin{array}{c}\text { Bag et al. }{ }^{6}(2012) ; \text { Chandrasekaran } \\
\& \text { Venkatesalu } 24(2004) ; \text { Yadav et } \\
\text { al. }{ }^{42} \text { (2011); Mariselvam et al. }{ }^{45} \\
\text { (2017) } \\
\text { Saha et al. }{ }^{25}(2013) \text {; Jasmine } \text { et al. } \\
\text { } 44 \text { (2010) } \\
\text { Chandrasekaran \& Venkatesalu }{ }^{24} \\
\text { (2004) } \\
\text { Bag et al. }{ }^{6} \text { (2012); Jasmine et al. }{ }^{44} \\
\text { (2010) }\end{array}$ \\
\hline & Klebsiella pneumoniae & $\begin{array}{l}\text { Agar diffusion } \\
\text { Disk diffusion } \\
\text { Microdilution } \\
\text { Macrodilution }\end{array}$ & $\begin{array}{c}\text { Bag et al. }{ }^{6}(2012) ; \text { Chandrasekaran } \\
\text { \& Venkatesalu }{ }^{24}(2004) \\
\text { Duraipandiyan } \text { et al. }{ }^{4}(2006) ; \\
\text { Jasmine } \text { et al. } \\
\text { Bag et al. }{ }^{6}(2012) ; \text { Jasmine } \text { et al. }{ }^{44} \\
\text { (2010) } \\
\text { Chandrasekaran \& Venkatesalu }{ }^{24} \\
\text { (2004) }\end{array}$ \\
\hline & $\begin{array}{l}\text { Pseudomonas } \\
\text { aeruginosa }\end{array}$ & $\begin{array}{l}\text { Agar diffusion } \\
\text { Disk diffusion } \\
\text { Macrodilution } \\
\text { Microdilution }\end{array}$ & $\begin{array}{c}\text { Bag et al. }{ }^{6} \text { (2012); Chandrasekaran } \\
\text { \& Venkatesalu }{ }^{24} \text { (2004);Yadav et al. } \text {. } \\
42 \text { (2011); Mariselvam et al. } 45 \text { (2017) } \\
\text { Jasmine et al. } 44 \text { (2010) } \\
\text { Chandrasekaran \& Venkatesalu }{ }^{24} \\
\text { (2004) } \\
\text { Bag et al. }{ }^{6} \text { (2012); Jasmine et al. }{ }^{44} \\
\text { (2010) }\end{array}$ \\
\hline & Staphylococcus aureus & $\begin{array}{l}\text { Agar diffusion } \\
\text { Disk diffusion } \\
\text { Macrodilution } \\
\text { Microdilution }\end{array}$ & $\begin{array}{c}\text { Bag et al. }{ }^{6}(2012) ; \text { Chandrasekaran } \\
\text { \& Venkatesalu } 24(2004) ; \text { Yadav et } \\
\text { al. }{ }^{24}(2011) ; \\
\text { Duraipandiyan et al. }{ }^{4}(2006) \\
\text {;Saha } \text { et al. }{ }^{25}(2013) \\
\text { Chandrasekaran \& Venkatesalu }{ }^{24} \\
\text { (2004) } \\
\text { Bag et al. }{ }^{6}(2012)\end{array}$ \\
\hline & Streptococcus aureus & Agar diffusion & Mariselvam et al. 45 (2017) \\
\hline $\begin{array}{l}\text { Stem } \\
\text { bark }\end{array}$ & $\begin{array}{c}\text { Bacillus subtilis; } \\
\text { Escherichia coli; } \\
\text { Klebsiella pneumonia; } \\
\text { Staphylococcus aureus }\end{array}$ & Agar diffusion & Prabhakaran et al. ${ }^{46}$ (2011) \\
\hline Root & $\begin{array}{c}\text { Streptococcus } \\
\text { mutans;Streptococcus } \\
\text { oralis }\end{array}$ & Microdilution & Cartaxo-Furtado et al. ${ }^{47}$ (2015) \\
\hline
\end{tabular}

Table 3. Evidence of studies demonstrating antifungal activity of Syzygium cumin extracts obtained from different parts of the plant against fungi of the genus Candida.

\begin{tabular}{|c|c|c|c|}
\hline $\begin{array}{l}\text { Plant } \\
\text { parts }\end{array}$ & Microorganisms & $\begin{array}{c}\text { Antimicrobial } \\
\text { susceptibility test }\end{array}$ & References \\
\hline \multirow[t]{2}{*}{ Fruits } & Candida albicans & $\begin{array}{l}\text { Agar dilution } \\
\text { Disk diffusion } \\
\text { Microdilution }\end{array}$ & $\begin{array}{l}\text { Shad et al. }{ }^{26}(2014) \\
\text { Saha et al. }{ }^{25}(2013) \\
\text { Migliato et al. }{ }^{27}(2010)\end{array}$ \\
\hline & $\begin{array}{c}\text { Candida krusei; } \\
\text { Candida parapsilosis }\end{array}$ & Microdilution & Migliato et al. ${ }^{27}$ (2010) \\
\hline $\begin{array}{l}\text { Fruit } \\
\text { peel }\end{array}$ & Candida albicans & $\begin{array}{l}\text { Disk diffusion; } \\
\text { Macrodilution }\end{array}$ & Priya et al. ${ }^{88}$ (2013) \\
\hline \multirow[t]{5}{*}{ Leaves } & Candida albicans & $\begin{array}{l}\text { Agar diffusion } \\
\text { Disk diffusion } \\
\text { Microdilution }\end{array}$ & $\begin{array}{c}\text { Oliveira et al. }{ }^{8}(2007) \text {; Elfadil et al. } \\
\text { 35 (2015); Jadhav et al. } .^{8}(2015) ; \\
\text { Khan et al. } 49(2017) \\
\text { Bhargava et al. } 3^{0}(2009) \\
\text { Pereira } \text { et al. } 9 \text { (2009); Costa et al. }{ }^{21} \\
\text { (2009); Jadhav et al. } 3^{8}(2015) ; \\
\text { Pereira et al. } 4^{8}(2016) ; \text { Khan et al. } 49 \\
\text { (2017) }\end{array}$ \\
\hline & Candida glabrata & $\begin{array}{l}\text { Agar diffusion } \\
\text { Microdilution }\end{array}$ & $\begin{array}{c}\text { Kaneria \& Chanda }{ }^{11}(2011) \text {; Khan et } \\
\text { al. } 49(2017) \\
\text { Costa et al. }{ }^{21}(2009) ; \text { Khan et al. }{ }^{49} \\
\text { (2017) }\end{array}$ \\
\hline & Candida krusei & $\begin{array}{l}\text { Agar diffusion } \\
\text { Disk diffusion } \\
\text { Microdilution }\end{array}$ & $\begin{array}{c}\text { Oliveira et al. }{ }^{8}(2007) ; \text { Khan et al. } \\
49(2017) \text {. } \\
\text { Bhargava et al. } \text { 30 }^{\circ}(2009) \\
\text { Khan et al. }{ }^{49}(2017)\end{array}$ \\
\hline & Candida neoformans & $\begin{array}{l}\text { Agar diffusion } \\
\text { Microdilution }\end{array}$ & $\begin{array}{c}\text { Kaneria \& Chanda } 31(2011) ; \text { Braga } \\
\text { et al. } 5^{50}(2007) \\
\text { Braga et al. } 5^{50}(2007)\end{array}$ \\
\hline & Candida tropicalis & $\begin{array}{l}\text { Agar diffusion } \\
\text { Microdilution }\end{array}$ & $\begin{array}{c}\text { Khan et al. }{ }^{49}(2017) \\
\text { Costa et al. }{ }^{21}(2009) ; \text { Khan et al. } 49 \\
\text { (2017) }\end{array}$ \\
\hline \multirow[t]{2}{*}{ Seeds } & Candida albicans & $\begin{array}{l}\text { Agar diffusion; } \\
\text { Macrodilution } \\
\text { Disk diffusion } \\
\text { Microdilution }\end{array}$ & $\begin{array}{c}\text { Chandrasekaran \& Venkatesalu }{ }^{24} \\
\text { (2004) } \\
\text { Saha et al. } 25 \text { (2013) } \\
\text { Höfling et al. }{ }^{2}(2010)\end{array}$ \\
\hline & $\begin{array}{c}\text { Candida dubliniensis; } \\
\text { Candida glabrata; } \\
\text { Candida guilliermondii; } \\
\text { Candida } \\
\text { krusei;Candida } \\
\text { lusitaniae; Candida } \\
\text { parapsilosis; Candida } \\
\text { rugosa; Candida } \\
\text { tropicalis; Candida } \\
\text { utilis }\end{array}$ & Microdilution & Höfling et al. ${ }^{2}(2010)$ \\
\hline \multirow[t]{2}{*}{$\begin{array}{l}\text { Stem } \\
\text { bark }\end{array}$} & Candida albicans & $\begin{array}{l}\text { Disk diffusion } \\
\text { Microdilution }\end{array}$ & $\begin{array}{l}\text { Prabhakar et al. }{ }^{51}(2008) \\
\text { Cartaxo-Furtado et al. }{ }^{47}(2015)\end{array}$ \\
\hline & $\begin{array}{c}\text { Candida glabrata; } \\
\text { Candida guilliermondii; } \\
\text { Candida krusei; } \\
\text { Candida parapsilosis; } \\
\text { Candida tropicalis }\end{array}$ & Disk diffusion & Prabhakar et al. ${ }^{51}$ (2008) \\
\hline
\end{tabular}




\section{DISCUSSION}

Native to tropical regions, Syzygium cumini is found in several countries of the Asian, African and American continent $t^{1,3,10,27}$. The plant has recognized medicinal potential, including antidiabetic activity ${ }^{10,11,18,27}$ and different other therapeutic purposes attributed to different pharmacological actions that can be obtained from parts of the plant such as seeds, leaves, stem and fruits ${ }^{1,3,6,10-13,15-18,27,31,35,50}$. In addition, its medicinal applications also extend to the use of different parts for curative purposes of oral affections ${ }^{1,10,14,19-22,24}$, with the demonstration of antibacterial and antifungal effects on oral microorganisms as corroborated by the literature findings mentioned earlier and showing the potencial applications of this plant to prevent and/or treat oral affections.

Within this context, the growing problem of microbial resistance to antibacterial and antifungal drugs ${ }^{2,4,6,9,21,22,27,31,37,44}$ highlights the need for identifying alternative sources of antimicrobials 4,6,21,22,27,31,37. Considering the importance of medicinal plants among these new antimicrobial agents $4,6,21,22,37$, the finding of antimicrobial activity of $S$. cumini extracts is therefore an interesting pharmacological property among the various other biological activities associated with its use.

The promising medicinal properties associated with the extracts obtained from different parts of $S$. cumini can be explained by the presence of different groups of bioactive compounds $^{10-12,16,22,33}$. For example, phytochemical compounds such as flavonoids, phenolics, tannins and saponins ${ }^{46}$ have been detected in the stem bark of the plant. Furthermore, the leaves contain secondary metabolites such as alkaloids ${ }^{5,22}$ anthraquinones $^{37}$, flavonoids ${ }^{10,22,31,37}$, glycosides $^{22}$, phenolics $^{22,31,46}$, phlobatannins $5^{5}$, saponins $^{5,22,46}$ steroids ${ }^{5,22}$, 'tannins $s^{5,10,22,37,46}$, triterpenoids $s^{5,10,37}$ and terpenoids ${ }^{22}$. The fruits of S. cumini contain alkaloids ${ }^{26}$, anthocyanins ${ }^{10}$, anthocyanidins $^{28}$, flavonoids ${ }^{25,26}$, phenolics ${ }^{25,26}$, saponins, steroids and tannins ${ }^{26}$, while the seeds contain chemical compounds such as alkaloids $^{6,10,45,46,52}, \quad$ flavonoids $^{6,10,25,44,46,52}$, glycosides ${ }^{6,10,44,52}$, proanthocyanidins, monosaccharides $^{52}$, reducing sugars ${ }^{44,45,52}$, saponins $^{6,44,52}$, steroids ${ }^{44,52}$, tannins ${ }^{45,46}$ and terpenoids ${ }^{6}$. Finally, the roots have been shown to contain alkaloids, flavonoids, phenols and tannins ${ }^{46}$. Hence, since anthocyanins ${ }^{2}$, flavonoids $^{2,11,22,33}$, phenolic compounds $s^{11,31,53}$, saponins $^{33}$, steroids ${ }^{22,54}$ and tannins $s^{2,11,33,50}$ have antimicrobial effects and secondary metabolites such as flavonoids ${ }^{55,56}$, phenolic compounds ${ }^{31}$, saponins $^{55,57}$, tannins ${ }^{57}$ and terpenes ${ }^{55}$ exert antifungal effects, the existence of antimicrobial and antifungal activities of the extracts obtained from different parts of $S$. cumini can be justified, with these activities being corroborated by the findings of the studies mentioned earlier.

Thus, the antimicrobial effects on pathogenic microorganisms such as Streptococcus mutans ${ }^{9,33}$, other bacteria of the genus Streptococcus ${ }^{47}$, fungi of the genus Candida $a^{2,9,47}$ and microorganisms associated with endodontic and periapical infections such as Enterococcus faecalis, Escherichia coli and Staphylococcus aureus 9 , as well as microorganisms associated with infections that can potentially affect the oral cavity, such as Neisseria gonorrhoeae ${ }^{38}$ and Bacillus subtilis ${ }^{9,43}$, reinforce the promising medicinal effect of the $S$. cumini extract, indicating possible applications to the treatment of affections of the oral cavity like dental caries, periodontal disease, endodontic infections and oral candidiasis ${ }^{9}$. In addition, other biological activities such as antiinflammatory, antioxidant and anticarcinogenic effects $^{16}$ indicate a vast and promising therapeutic potential of the extracts of this plant that go beyond the antibacterial and antifungal effects highlighted here.

CONCLUSION

The present results permit us to state that, in addition to the popular use of different parts of S. cumini for therapies of interest and/or for application to Dentistry, the evidence of the antibacterial and antifungal effects of their extracts on different pathogenic oral microorganisms or those that can potentially affect this site, indicates that they present a potential therapeutic activity and may be used in Dentistry for the prevention and/or treatment of oral affections.

\section{REFERENCES}

1. Migliato KF, Baby AR, Zague V, Velasco MVR, Corrêa MA, Sacramento LVS, et al. Ação farmacológica de Syzygium cumini (L.) Skeels. Acta Farm Bonaerense 2006;25(2): 310-14.

2. Höfling JF, Anibal PC, Obando-Pereda GA, Peixoto IA, Furletti VF, Foglio MA et al. Antimicrobial potential of some plant extracts against Candida species. Braz. J. Biol. 2010; 70(4):1065-68

3. Migliato KF, Moreira RRD, Mello JCP, Sacramento LVS, Corrêa MA, Salgado HRN. Controle da qualidade do fruto de Syzygium cumini (L.) Skeels. Rev bras farmacogn. 2007;17(1):94-101. 
4. Duraipandiyan V, Ayyanar M, Ignacimuthu S. Antimicrobial activity of some ethnomedicinal plants used by paliyar tribe from Tamil Nadu, India. BMC Complementary and Alternative Medicine 2006; 6:35

5. Kaneria M, Baravalia Y, Vaghasiya Y, Chanda S. Determination of antibacterial and antioxidant potential of some medicinal plants from saurashtra region, India. Indian J Pharm Sci. 2009;71(4):406-12.

6. Bag A, Bhattacharyya SK, Pal NK, Chattopadhyay RR. In vitro antibacterial potential of Eugenia jambolana seed extracts against multidrug-resistant human bacterial pathogens. Microbiol Res. 2012;167(6):352-7.

7. Noldin VF, Cechinel Filho V, Monache FD, Benassi JC, Christmann IL, Pedrosa RC, et al. Composição química e atividades biológicas das folhas de Cynara scolymus L. (alcachofra) cultivada no Brasil. Quím. Nova 2003; 26(3):331-334.

8. Oliveira GF, Furtado NAJC, Silva-Filho AA, Martins CHG, Bastos JN, Cunha WR, et al. Antimicrobial activity of Syzygium cumini (Myrtaceae) leaves extract. Braz. J. Microbiol. 2007; 38(2):381-384.

9. Pereira CA, Vilela PGF, de Oliveira LD, Jorge AOC. Ação antimicrobiana in vitro de extratos glicólicos de Psidium guajava L., Syzygium cumini L. e Pimpinella anisum L. Rev Inst Adolfo Lutz 2009; 68(1):102-108.

10. Ayynamar M, Subash-Babu P. Syzygium cumini (L.) Skeels: A review of its phytochemical constituents and traditional uses. Asian Pac J Trop Biomed. 2012; 2(3):240-246.

11. Srivastava S, Chandra D. Pharmacological potentials of Syzygium cumini: a review. J Sci Food Agric. 2013;93(9):2084-93.

12. Chagas VT, França LM, Malik S, Paes AM. Syzygium cumini (L.) skeels: a prominent source of bioactive molecules against cardiometabolic diseases. Front Pharmacol. 2015;6:259.

13. Baliga MS, Fernandes S, Thilakchand KR, D'souza P, Rao S. Scientific Validation of the Antidiabetic Effects of Syzygium jambolanum DC (Black Plum), a Traditional Medicinal Plant of India. J Altern Complement Med. 2013;19(3):191-197.

14. Atale N, Chakraborty M, Mohanty S, Bhattacharya S, Nigam D, Sharma M, Rani V. Cardioprotective role of Syzygium cumini against glucose-induced oxidative stress in H9C2 cardiac myocytes. Cardiovasc Toxicol. 2013;13(3):278-89.

15. Ribeiro TG, Chávez-Fumagalli MA, Valadares DG, Franca JR, Lage PS, Duarte MC et al.Antileishmanial activity and cytotoxicity of Brazilian plants. Exp Parasitol. 2014;143:60-68.

16. Bitencourt PE, Cargnelutti LO, Stein CS,
Lautenchleger $R$, Ferreira LM, Sangoi $M$, Denardi L, Borges RM, Boligon A, Moresco RN, Cruz L, Zanette RA, Alves SH, Moretto MB. Nanoparticle formulation increases Syzygium cumini antioxidant activity in Candida albicansinfected diabetic rats. Pharm Biol. 2017;55(1):1082-88.

17. Priya SH, Prakasan N, Purushothaman J. Antioxidant activity, phenolic-flavonoid content and high-performance liquid chromatography profiling of three different variants of Syzygium cumini seeds: A comparative study. J Intercult Ethnopharmacol. 2017;6(1):107-14.

18. Ajiboye BO, Ojo OA, Akuboh OS, Abiola OM, Idowu O, Amuzat AO. Anti-Hyperglycemic and Anti-Inflammatory Activities of PolyphenolicRich Extract of Syzygium cumini Linn Leaves in Alloxan-Induced Diabetic Rats. J Evid Based Integr Med. 2018;23:2515690X18770630.

19. Loguercio AP, Battistin A, Vargas AC, Henzel A, Witt NM. Atividade antibacteriana de extrato hidro-alcoólico de folhas de jambolão (Syzygium cumini (L.) Skells). Cienc. Rural 2005;35(2):371-376.

20. Ulla A, Alam MA, Sikder B, Sumi FA, Rahman MM, Habib ZF, Mohammed MK, Subhan N, Hossain H, Reza HM. Supplementation of Syzygium cumini seed powder prevented obesity, glucose intolerance, hyperlipidemia and oxidative stress in high carbohydrate high fat diet induced obese rats. BMC Complement Altern Med. 2017;17(1):289.

21. Costa ACBP, Pereira CA, Freire F, Junqueira JC, Jorge AOC. Atividade antifúngica dos extratos glicólicos de Rosmarinus officinalis Linn. e Syzygium cumini Linn. sobre cepas clínicas de Candida albicans,Candida glabrata e Candida tropicalis. Rev odontol UNESP 2009; 38(2):111-16.

22. Gowri SS, Vasantha K. Phytochemical screening and antibacterial activity of Syzygium cumini (L.) (Myrtaceae) leaves extracts. Int.J. PharmTech Res. 2010; 2(2):1569-1573.

23. Borde VU, Pawar DP, Shelar SR, Apturkar RM. Antimicrobial activity of some medicinal plants. Science Research Reporter 2013; 3(1):33-37

24. Chandrasekaran $M$, Venkatesalu V. Antibacterial and antifungal activity of Syzygium jambolanum seeds. J Ethnopharmacol. 2004; 91(1):105-108.

25. Saha RK, Zaman NM, Roy P. Comparative evaluation of the medicinal activities of methanolic extract of seeds, fruit pulps and fresh juice of Syzygium cumini in vitro. Journal of Coastal Life Medicine 2013; 1(4):300-308.

26. Shad AA, Ahmad S, Ullah R, AbdEI-Salam NM, Fouad H, Ur Rehman N, Hussain H, Saeed W. Phytochemical and biological activities of four wild medicinal plants. ScientificWorldJournal. 2014;2014:857363. 
27. Migliato KF, Mello JCP, Higa OZ, Rodas ACD, Corrêa MA, Mendes-Giannini MJS, et al. Antimicrobial and cytotoxic activity of fruit extract from Syzygium cumini (L.) Skeels. Lat. Am. J. Pharm. 2010; 29(5):725-730

28. Priya SSL, Devi PR, Eganathan P, Kingsley J. In vitro antimicrobial activity of Syzygium cumini fruit peel and identification of anthocyanins. Afr. J. Pharm. Pharmacol. 2013;7(25):1719-1728

29. Shafi PM, Rosamma MK, Jamil K, Reddy PS. Antibacterial activity of Syzygium cumini and Syzygium travancoricum leaf essential oils. Fitoterapia 2002; 73(5):414-416.

30. Bhargava S, Bhargava P, Jain UK. Evaluation of ulcer-protective and antimicrobial activity of Syzygium cumini (linn.) Skeels leaves. Pharmacology online 2009;3:266-74.

31. Kaneria M, Chanda S. Evaluation of antioxidant and antimicrobial capacity of Syzygium cumini L. leaves extracted sequentially in different solvents. Journal of Food Biochemistry 2011; 37(2):168-176.

32. Elansary HO, Salem MZM, Ashmawy NA, Yacout MM. Chemical composition, antibacterial and antioxidant activities of leaves essential oils from Syzygium cumini L., Cupressus sempervirens L. and Lantana camara L. from Egypt. JAS. 2012;4(10):144152.

33. Tahir L, Ahmed S, Hussain N, Perveen I, Rahman S. Effect of leaves extract of indigenous species of Syzygium cumini on dental caries causing pathogens. Int J Pharm Bio Sci 2012; 3(3):1032-1038.

34. Mohamed AA, Ali SI, El-Baz FK. Antioxidant and antibacterial activities of crude extracts and essential oils of Syzygium cumini leaves.PLoS ONE 2013; 8(4):e60269.

35. Elfadil AG, Abualhassan AM, Hamid AA, Sabahelkhier MK. Antimicrobial activities of Syzygium cumini leave extracts against selected microorganisms. Nova Journal of Medical and Biological Sciences 2015; 4(2).

36. Bajracharya AM, Yami KD, Prasai T, Basnyat SR, Lekhak B. Screening of some medicinal plants used in Nepalese traditional medicine against enteric bacteria. Scientific World 2008; 6(6):107-110.

37. Bouzada MLM, Fabri RL, Nogueira M, Konno TUP, Duarte GG, Scio E. Antibacterial, cytotoxic and phytochemical screening of some traditional medicinal plants in Brazil. Pharm Biol. 2009;47(1):44-52.

38. Jadhav N, Kulkarni S, Mane A, Kulkarni R, Palshetker A, Singh K, Joshi S, Risbud A, Kulkarni S. Antimicrobial activity of plant extracts against sexually transmitted pathogens. Nat Prod Res. 2015;29(16):1562-6.

39. Viera TI, Gondim BLC, Santiago BM, Valença AMG. In vitro antibacterial and non-stick activity of extracts from leaves of Psidium guineense Sw. and Syzygium cumini (L.) Skeels on oral microorganisms. Rev Gaúcha Odontol 2012; 60(3):359-365.

40. Chanudom L, Bhoopong P, Khwanchuea R, Tangpong J. Antioxidant and antimicrobial activities of aqueous \& ethanol crude extracts of Thai Traditional Plants. Int J Curr Microbiol App Sci. 2014;3(1):549-58.

41. Pereira NLF, Aquino PEA, Júnior JGAS, Cristo JS, Vieira Filho MA, Moura FF, et al.Antibacterial activity and antibiotic modulating potential of the essential oil obtained from Eugenia jambolana in association with led lights. J Photochem Photobiol B. 2017; 174:144-149.

42. Yadav SS, Meshram GA, Shinde D, Patil RC, Manohar SM, Upadhye MV. Antibacterial and Anticancer Activity of Bioactive Fraction of Syzygium cumini L. Seeds. HAYATI Journal of Biosciences 2011; 18(3):118-122

43. Yadav AK, Saraswat S, Sirohi P, Rani M, Srivastava S, Singh MP et al. Antimicrobial action of methanolic seed extracts of Syzygium cumini Linn.on Bacillus subtilis. AMB Expr 2017; 7:196

44. Jasmine $R$, Selvakumar $B N$, Daisy $P$, Ignacimuthu S. Activity of Eugenia jambolana, an ethnomedical plant, against drug-resistant bacteria. Pharm Biol. 2010;48(4):405-10.

45. Mariselvam R, Ranjitsingh AJA, Selvakumar PM, Krishnamoorthy $R$, Alshatwi AA. Eco friendly natural dyes from Syzygium cumini (L) (Jambolan) fruit seed endosperm and to preparation of antimicrobial fabric and their washing properties.Fibers Polym 2017; 18:460

46. Prabhakaran S, Gothandam KM, Sivashanmugam K. Phytochemical and antimicrobial properties of Syzygium cumini an ethanomedicinal plant of Javadhu hills. Research in Pharmacy 2011; 1(1):22-32.

47. Cartaxo-Furtado NADEO, Sampaio TO, Xavier MA, Medeiros ADDE, Pereira JV. Perfil fitoquímico e determinação da atividade antimicrobiana de Syzygium cumini (L.) Skeels (Myrtaceae) frente a microrganismos bucais. Rev. bras. plantas med. 2015; 17(4,suppl.3):1091-1096.

48. Pereira JV, Freires IA, Castilho AR, da Cunha MG, Alves $\mathrm{H}$ da S, Rosalen PL. Antifungal potential of Sideroxylon obtusifolium and Syzygium cumini and their mode of action against Candida albicans. Pharm Biol. 2016; 54(10):2312-2319.

49. Khan S, Imran M, Imran M, Pindari N. Antimicrobial activity of various ethanolic plant extracts against pathogenic multi drug resistant Candida spp. Bioinformation. 2017;13(3):67-72.

50. Braga FG, Bouzada ML, Fabri RL, de O Matos M, Moreira FO, Scio E et al. 
Antileishmanial and antifungal activity of plants used in traditional medicine in Brazil. J Ethnopharmacol. 2007;111(2):396-402.

51.Prabhakar K, Kumar LS, Rajendran S, Chandrasekaran M, Bhaskar K, Sajit Khan AK. Antifungal Activity of Plant Extracts against Candida Species from Oral Lesions. Indian J Pharm Sci. 2008;70(6):801-3.

52. Atale N, Jaiswal A, Chhabra A, Malhotra U, Kohli S, Mohanty S, et al. Phytochemical and antioxidant screening of Syzygium cumini seed extracts: a comparative study. Journal of Pharmacy Research 2011; 4(12):4530-4532

53. Soobrattee M, Neergheen VS, Luximon-ramma A, Aruoma OI, Bahorun T. Phenolics as potential antioxidant therapeutic agents: mechanism and actions. Mutation Research 579;(2005):200-213.

54. Aiyelaagbe OO, Osamudiamen PM. Phytochemical screening for active compounds in Mangifera indica leaves from Ibadan, Oyo State. Plant Sci Res. 2009;2(1):11-13.

55. Morais MI, Pinto ME, Araújo SG, Castro AH, Duarte-Almeida JM, Rosa LH, Rosa CA, Johann S, Lima LA. Antioxidant and antifungal activities of Smilax campestris Griseb. (Smilacaceae). Nat Prod Res. 2014;28(16):1275-9.

56. Oliveira DM, Silva TFR, Martins MM, de Morais SAL, Chang R, de Aquino FJT, et al. Antifungal and cytotoxicity activities of Banisteriopsis argyrophylla leaves. J Pharm Pharmacol.2018; 70(11):1541-1552.

57. Salhi N, Mohammed Saghir SA, Terzi V, Brahmi I, Ghedairi N, Bissati S. Antifungal Activity of Aqueous Extracts of Some Dominant Algerian Medicinal Plants. Biomed Res Int. 2017;2017:7526291.

\section{CONFLICTS OF INTERESTS}

The authors declare no conflicts of interests.

CORRESPONDING AUTHOR

Ernani Canuto Figueirêdo Júnior

Postgraduate Program in Dentistry, State University of Paraíba, UEPB

58429-500 Campina Grande - PB, Brazil

E-mail: ernanicfjunior@outlook.com 